Dom. Cien., ISSN: 2477-8818

Vol. 3, núm. mon., agos., 2017, pp. 137-148

Cyberbullying: el acoso a través de las redes sociales en jóvenes universitarios

\title{
Cyberbullying: el acoso a través de las redes sociales en jóvenes universitarios
}

\author{
Cyberbullying: harassment through social networks in young university students
}

\section{Cyberbullying: assédio através de redes sociais em jovens estudantes universitários}

'Tania C. Cañarte-Rodríguez

taniacanarte2016@gmail.com

Recibido: 27 de enero de 2017 * Corregido: 20 de abril de $2017 *$ Aceptado: 5 de julio de 2017

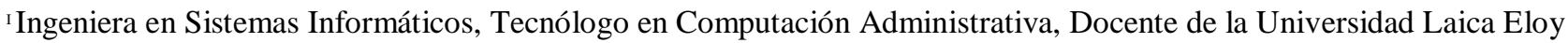
Alfaro de Manabí, Manta, Ecuador en Sistemas, Universidad Laica Eloy Alfaro de Manabí, Manta, Ecuador. 


\section{Resumen}

Se efectuó una investigación cualitativa, descriptiva y transversal, en los estudiantes de la Facultad de Informática de la Universidad Laica "Eloy Alfaro" de Manabí, en la ciudad de Manta, República de Ecuador, durante el periodo 2015, a fin de determinar las experiencias de victimización a través de las nuevas tecnología en jóvenes universitarios. La población de estudio estuvo conformada por 50 jóvenes. El análisis se llevó a cabo a partir de categorías en lugar de variables dependientes e independientes. En la serie se obtuvo que el $80.0 \%$ de las mujeres plantearon haber sido molestadas o acosadas por internet, el $60.0 \%$ en los hombres; actos de violencia por medio de las nuevas tecnologías que prevaleció fue el insulto, el $100 \%$ de los cyber acosados han sido en algún momento molestados por medio del internet como insultos, acoso sexual. Infortunadamente, el acoso y el cyber acoso son conductas violentas que se han naturalizado. El uso de redes sociales, como forma de interacción humana, ha supuesto una prolongación de actividades delictivas. Es necesaria la realización de investigaciones, las cuales busquen estrategias de intervención frente a este fenómeno de violencia.

Palabras clave: jóvenes; redes sociales; cyber acoso.

\section{Abstract}

A qualitative, descriptive and transversal investigation was carried out in the students of the Faculty of Informatics of the "Eloy Alfaro" Laica University of Manabí, in the city of Manta, Republic of Ecuador, during the period 2015, in order to determine the experiences Of victimization through the new technology in young university students. The study population consisted of 50 young people. The analysis was carried out from categories rather than dependent and independent variables. In the series, $80.0 \%$ of the women reported having been harassed or harassed by the Internet, $60.0 \%$ in men; Acts of violence through the new technologies that prevailed was the insult, $100 \%$ of harassed cyber have been at one time molested through the internet as insults, sexual harassment. Unfortunately, harassment and cyber bullying are violent behaviors that have become naturalized. The use of social networks, as a form of human interaction, has meant an extension of criminal activities. It is necessary to conduct research, which seeks strategies for intervention in the face of this phenomenon of violence.

Keywords: young; social networks; cyber bullying. 


\section{Resumo}

Uma investigação qualitativa, descritiva e transversal foi realizada nos estudantes da Faculdade de Informática da Universidade Eica Alfaro, Laica de Manabí, na cidade de Manta, República do Equador, no período de 2015, para determinar as experiências De vitimização através da nova tecnologia em jovens estudantes universitários. A população estudada era composta por 50 jovens. A análise foi realizada a partir de categorias, em vez de variáveis dependentes e independentes. Na série, $80,0 \%$ das mulheres relataram ter sido assediado ou assediado pela internet, $60,0 \%$ em homens; Os atos de violência através das novas tecnologias que prevaleceram foi o insulto, 100\% do ciber assediado foram simultaneamente molestados pela internet como insultos, assédio sexual. Infelizmente, o assédio e o bullying cibernético são comportamentos violentos que se tornaram naturalizados. $\mathrm{O}$ uso das redes sociais, como forma de interação humana, significou uma extensão das atividades criminosas. É necessário realizar pesquisas, que buscam estratégias de intervenção diante desse fenômeno de violência.

Palavras chave: jovem; redes sociais; bullying da internet

\section{Introducción}

El concepto de red social ha adquirido una importancia notable en los últimos años. Se ha convertido en una expresión del lenguaje común que asociamos a nombres como Facebook o Twitter. Pero su significado es mucho más amplio y complejo. Las redes sociales son, desde hace décadas, objeto de estudio de numerosas disciplinas. Alrededor de ellas se han generado teorías de diverso tipo que tratan de explicar su funcionamiento y han servido, además, de base para su desarrollo virtual. Con la llegada de la Web 2.0, las redes sociales en Internet ocupan un lugar relevante en el campo de las relaciones personales y son, asimismo, paradigma de las posibilidades que nos ofrece esta nueva forma de usar y entender Internet (Redes Sociales. 2014).

Las redes sociales se organizan en base a categorías, que pueden ser relacionadas con el trabajo, el ocio u otros intereses de los usuarios. Por ejemplo, Facebook nos muestra el número de amigos/as y Linkedin categoriza los perfiles en función de su ocupación, lo que favorece el encuentro entre profesionales (Redes Sociales. 2014). 
En sentido amplio, una red social es una estructura social formada por personas o entidades conectadas y unidas entre sí por algún tipo de relación o interés común.

La proliferación de los ordenadores y de los servicios de internet significa que hay millones de menores y adolescentes de todo el mundo que tienen acceso a páginas web sin supervisión alguna. El acceso a la información es sin duda una excelente herramienta para los estudiantes que investigan temas relacionados con sus deberes escolares. No obstante, muchas familias y profesores se sienten alarmados ante el influjo negativo de internet. Por ejemplo, tanto los niños como los adolescentes que chatean en línea con sus conocidos han sido atraídos a relaciones cibersexuales y a veces a reunirse con compañeros adultos de chateo que los explotan (Curry, 2000; Donnerstein y Smith, 2001).

Los procesos sociales, culturales y económicos de la sociedad contemporánea están impactados por las Tecnologías de la Información y Comunicación (TIC), pero también por los aspectos íntimos de las personas. Estos impactos están generando una cultura de lo virtual, del ciberespacio, de una cbercultura a la que los jóvenes se incorporan y así, generan estilos de vida en los que la tecnología es parte de su vida cotidiana, pues a través de ella se transmiten formas de pensar, de ser, de emocionarse y de comportarse; los jóvenes simultáneamente comparten su vida cotidiana y el entretenimiento. A través de los diferentes accesorios digitales se determinan diversas realidades juveniles, que de alguna manera han contribuido a la definición o reafirmación de percepciones, comportamientos y actitudes de la propia identidad, incluyendo la identidad de género, que en ocasiones propicia violencia simbólica o entornos agresivos (Serrano Barquín1 C 2014).

Desde el comienzo de la civilización, la violencia se ha manifestado de diversas formas, siendo su impronta una clave importante para entender las relaciones humanas. Este fenómeno no es nuevo, pero sí el ámbito de su reproducción, la forma exponencial en que se manifiesta y sus inesperados efectos. Las TIC y su presuroso avance han transformado las formas de convivencia e interacción (Serrano Barquín1 C 2014).

Este espacio virtual favorece el acceso a distintas personalidades que los jóvenes adquieren a partir del uso de máscaras virtuales, éstas, permiten aflorar, fomentar o confrontar identidades de género. Por lo tanto, se asume que en las redes sociales generalmente se reportan identidades fragmentarias que sin lugar a dudas resulta interesante estudiar y conocer desde la perspectiva de los estudios de género, para así ofrecer actitudes reflexivas y en su caso propositivas (Serrano Barquín1 C 2014). 
Sin participación en redes sociales y sin mensajes, el ser joven resulta hoy impensable, sobre todo si se trata de jóvenes urbanos con cierto nivel socioeconómico. El anclaje en un grupo de pares, el estar accesible para los amigos, se sustenta en gran medida en la comunicación intragrupal, lo cual además ayuda a diferenciarse del mundo de los adultos a partir, entre muchas otras cosas, de la creación de “idolectos digitales o electrónicos" particulares. La participación en las redes sociales parece implicarles a los y las jóvenes un elemento de integración social, de asociación y de relación entre pares. Sin embargo, también ha trastocado en cierta forma su propia identidad ya que para los y las usuarios de las redes sociales existe una distinción entre sus "yo" reales y sus "yo" virtuales (Serrano Barquín1 C 2014).

Cuando se utilizan las redes sociales como medio para la agresión, el fenómeno se denomina cyberbullying. Una de las manifestaciones más frecuentes de este fenómeno es la publicación de fotografías, casi siempre poco afortunadas, que pueden ocasionar molestia a sus protagonistas, a los que se suele etiquetar para que sus contactos vean las imágenes. Es también muy frecuente la creación de páginas o grupos destinados a agredir, burlar o denunciar algún aspecto íntimo de la víctima.

En la actualidad, el cyberbullying resulta relevante por la gravedad de sus consecuencias, la dificultad de prevención y el alto grado de prevalencia (Cyberbullying: el acoso a través de las redes sociales. 2017).

Con el presente trabajo se pretendió determinar las experiencias de victimización a través de las nuevas tecnología en jóvenes universitarios.

\section{Metodología}

Se efectuó una investigación cualitativa, descriptiva y transversal, en los estudiantes de la Facultad de Informática de la Universidad Laica "Eloy Alfaro" de Manabí, en la ciudad de Manta, República de Ecuador, durante el periodo 2015, a fin de determinar las experiencias de victimización a través de las nuevas tecnología en jóvenes universitarios comprendidos entre las edades 17-20 años.

La muestra se obtuvo mediante el muestreo no probabilístico (no aleatorio) $\mathrm{n}=50$

El análisis se llevó a cabo a partir de categorías en lugar de variables dependientes e independientes, al tratarse de una investigación cualitativa, dado que "tiene el interés de comprender la realidad dentro 
de un contexto dado, por ello debe captarla como un todo unificado, no puede fragmentarse, no puede dividirse en variables independientes y dependientes" (Picaso Mesén M 2002).

El instrumento utilizado fue una encuesta la cual fue elaborada por el autor. La misma contiene datos como demográficos, otros relacionados con la experiencia de victimización y otros elementos de importancia para la investigación. Para facilitar el trabajo y la recogida de información, las posibles respuestas a cada pregunta fueron todas cerradas.

Experiencia de victimización se le pregunto sobre la experiencia de la victimización a través de las nuevas tecnología que hubieran podido sufrir los encuestados en algún momento, acoso, divulgación de videos, fotografías personales con ánimo de maldad, las veces que ocurrió dicha situación. Finalmente se preguntó cuál sería el motivo de haber sido acosados.

Otro dato de importancia sobre el ciberbullying, es que las preguntas fueron relacionadas al medio que ellos creen que es utilizado con mayor frecuencia victimizar a través de las nuevas tecnologías.

Los datos recogidos del instrumento fueron introducidos en una base de datos creada para tal efecto y posteriormente procesados a través del programa estadístico SPSS.

Se calcularon las medidas de frecuencia para variables cualitativas (absolutas y relativas) y se utilizó el porcentaje como medida de resumen.

\section{Resultados y discusiones}

En relación con el sexo de los encuestados tal como se puede observar en la tabla 1 del total de población en estudio (50) $70.0 \%$ son femeninos y $30.0 \%$ pertenecen al sexo masculino.

\begin{tabular}{|l|l|l|}
\hline Sexo & No & \% \\
\hline Masculino & 15 & 30.0 \\
\hline Femenino & 35 & 70.0 \\
\hline Total & $\mathbf{5 0}$ & $\mathbf{1 0 0}$ \\
\hline
\end{tabular}

El internet se ha convertido en pocos años, en una revolución tecnológica de la cual nadie duda de las ventajas que nos aporta como forma de comunicación global. Las posibilidades que la red nos ofrece 
son ilimitadas, de tal forma que han cambiado nuestra forma de relacionarnos, de trabajar y de pasar nuestro tiempo de ocio (Verdejo Espinosa M A 2015).

El uso de redes sociales, como forma de interacción humana, ha supuesto una prolongación de actividades delictivas que en la mayoría de los casos ya existían, pero en su variante digital, amplificadas por las peculiaridades de las comunicaciones a través de la red, como son la sencillez para conseguir anonimato, o el hecho de poder suplantar fácilmente cualquier dato que vincule a una identidad, ya sea perfil, dirección de correo, imagen, etc (Verdejo Espinosa M A 2015).

Un estudio realizado en 2010, preguntaba a las y los adolescentes si habían utilizado Internet para perjudicar a alguien (con el envío de fotos, vídeos o comentarios), o si habían sido perjudicadas o perjudicados por alguien, encontrando diferencias en cuanto a que las chicas se percibían más víctimas que los chicos y ellos reconocían en mayor proporción haber sido perpetradores de ese comportamiento (La tecnología en la pre adolescencia y adolescencia. 2010).

Tanto hombres como mujeres se ven afectados por el ciberacoso, pero un estudio realizado en India mostró que entre las víctimas de entre 18 y 32 años predominan las mujeres (El uso de la tecnología para perpetrar la violencia hacia las mujeres. 2010).

Al aplicar la encuesta a los participantes en la investigación en relación a la pregunta si alguien a usado el internet para molestarlo o acosarlo el $80.0 \%$ de las mujeres respondieron positivamente (28) en un menor porciento los varones $60.0 \%$ (9).

Tabla 2. Alguna vez alguien ha usado el internet para molestarte o acosarte.

\begin{tabular}{|l|c|c|c|c|}
\hline Sexo & Si & \% & No & \% \\
\hline Masculino & 9 & 60 & 6 & 40 \\
\hline Femenino & 28 & 80 & 7 & 20 \\
\hline
\end{tabular}

Respecto a la pregunta ¿Alguna vez alguien ha usado internet para divulgar o hacer llegar a la gente cosas malas de ti? los resultados fueron similar a la interrogante anterior el mayor porciento estuvo en las mujeres $16(45.0 \%)$ y en los varones $4(26.0 \%)$. 
En cuanto a la pregunta ¿Alguna vez alguien ha usado el internet para divulgar fotos o videos suyos? $34.2 \%$ de las encuestadas han vivido dicha experiencia. Su investigación Buldu (2014), plantea que estadísticamente se ha encontrado que esta experiencia está relacionada con el sexo.

Según los autores (Bernal y Angulo, 2013:5) "en su estudio el sentido y significado de la importancia de gestionar su privacidad y seguridad viene asociada a situaciones de acoso y abuso principalmente, pues aunque conocen los peligros de que te roben información valiosa, o de que te puedan piratear la clave y suplantarte, etc., lo primero lo ven como una posibilidad lejana".

Bartrina Andrés (2014), plantea que el tipo de acoso electrónico más habitual que practican los menores son las amenazas, los insultos, las injurias y las coacciones, todas ellas acciones contra el honor y la libertad personal y la integridad.

Como se muestra en la tabla 3 relacionado con los tipos de acoso por medio de las nuevas tecnologías, el insulto fue la situación que mayor prevaleció en los encuestados siendo en número mayor en las mujeres 12 (34.2\%), le continuó seguimiento mediante software espía (25.7\%). En el estudio realizado por Buldu A (2014), prevaleció los insultos ofensivos en un por ciento mayor en los varones.

Otro estudio realizado por Bartrina Andrés (2014), predominaron en los casos de ciberacoso (ciberbullying), la conexión con el tipo de acoso tradicional (bullying), tipos de acciones contra la integridad y contra el honor y la libertad personal, así como la combinación de ambos.

Según un estudio realizado para APC en Pakistán, en 2009 cerca de 94\% de las encuestadas declaró haber recibido llamadas y mensajes de acoso de parte de hombres que conocían

Tabla 3. Alguna vez ha vivido algún tipo de acoso actos planteados en el estudio.

\begin{tabular}{|l|c|c|c|}
\hline Tipos de acoso & Masculino & Femenino & Total \\
\hline $\begin{array}{l}\text { Distribución de fotos } \\
\text { de carácter sexual } \\
\text { sobre su persona }\end{array}$ & 6 & 8 & 14 \\
\hline Insultos & 3 & 12 & 15 \\
\hline $\begin{array}{l}\text { Seguimiento } \\
\text { mediante un software } \\
\text { espía }\end{array}$ & 3 & 9 & 12 \\
\hline
\end{tabular}


Dom. Cien., ISSN: 2477-8818

Vol. 3, núm. mon., agos., 2017, pp. 137-148

Cyberbullying: el acoso a través de las redes sociales en jóvenes universitarios

\begin{tabular}{|l|c|c|c|}
\hline Videos denigrantes & 2 & 1 & 3 \\
\hline $\begin{array}{l}\text { Promoción de } \\
\text { rumores difamatorios }\end{array}$ & 1 & 5 & 6 \\
\hline Total & $\mathbf{1 5}$ & $\mathbf{3 5}$ & $\mathbf{5 0}$ \\
\hline
\end{tabular}

Del total de los encuestados, también se indago si en algún momento el acosado, fue ciberacosador, respondieron a la misma positivamente 5 varones $(33.3 \%)$ y 3 mujeres $(8.5 \%)$. En su investigación Buldu A (2014), plantea que una persona que ha sido molestada o acosada a través de las nuevas tecnologías, tiene más probabilidades de ser ciberacosador.

El $100 \%$ de los ciberacosadores han sido en algún momento molestados por medio del internet como insultos, acoso sexual.

Al preguntar acerca de cuáles son las conductas de victimización llevadas a cabo, la distribución de videos o foto de carácter sexual representó el mayor porcentaje.

Con respecto al medio utilizado con más frecuencia para acosar, se observa en el grafico que más de la mitad de los encuestados, plantean que las victimizaciones se producen por Facebook. Estos resultados no coinciden con el estudio de Buldu A (2014).

Grafico. Medios electrónicos más utilizados para acosar

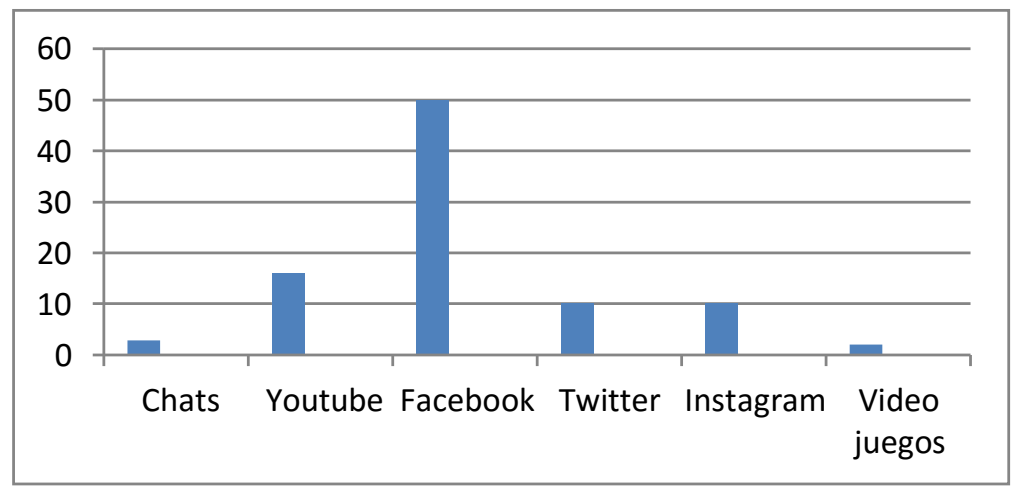

Se plantea en este momento, la necesidad de profundizar en estudios de victimización desde una vertiente más cualitativa, como también la necesidad de formar a los profesionales en competencias tecnológicas, con el fin de capacitarlos para que puedan resolver estos tipos de conflictos. Es muy importante también que se divulguen protocolos y líneas de actuación que regulen y eviten la 
arbitrariedad de la intervención frente a este tipo de situaciones y problemáticas emergentes en el ámbito juvenil (Bartrina Andrés, M J 2014).

De cara a la prevención de este fenómeno son buenas las actuaciones que pueden surgir buenos resultados. Destaca Capaldi (2004), la interacción social relacionada con las estructuras positivas de involucración parental de acompañamiento y disciplina, se relacionan con la ausencia de problemas, y por el contrario, el rechazo de los iguales pro sociales y la asociación con jóvenes involucrados en comportamientos problemáticos se consideran factores de riesgo, pues es con estos jóvenes con quienes muchos otros problemas de comportamiento se inician, se practican y se mantienen.

En cuanto a la conducta de prevención, la mayoría de los encuestados respondieron que la intervención de los padres y familiares, es la que mejor funciona (78.0 \%), seguida por la ayuda por parte de los amigos y de otros profesionales $(16.0 \%)$. El resto de los encuestados no presentó criterio alguno $(6.0 \%)$.

Finalmente, en relación al malestar que les ha producido ser víctima a través de las nuevas tecnologías de algunos de los tipos de acoso planteados en la investigación, más de la mitad de los encuestados plantea que la victimización sufrida fue de poco gravedad (86.0\%), mientras que un porciento menor plantea que el hecho fue muy grave $(14.0 \%)$.

\section{Conclusiones y discusión}

Infortunadamente, el acoso y el ciberacoso son conductas violentas que se han naturalizado. El uso de redes sociales, como forma de interacción humana, ha supuesto una prolongación de actividades delictivas. Es necesaria la realización de investigaciones, las cuales busquen estrategias de intervención frente a este fenómeno de violencia.

\section{Referencias bibliográficas}

Buldu A (2014). El ciberoacoso. Una aproximación criminalística. Recuperado el 12 de junio de 2016. Disponible en https://repositori.upf.edu/bitstream/handle/10230/22822/E1\%20ciberacoso-TFM.pdf

BERNAL, César y ANGULO, Félix (2013). "Interacciones de los jóvenes andaluces en las redes sociales" en Comunicar, n. ${ }^{\circ} 40$ v. XX, Revista Científica de Educomunicación; páginas 25-30. 
Bartrina Andrés, M J 2014. Conductas de ciberacoso en niños y adolescentes. Hay una salida con la educación y la conciencia Social EDUCAR, 50 (2), pp. 383-400 Universitat Autònoma de Barcelona Barcelona, España.

Capaldi. (2004) A Preventing Violence and Releated Heath-Risking Social Behaviors in Adolescents: An NIH State of the Science Conference US Department of Health and Human Sevices Pp 49-52

Curry, A. (2000). Internet Use in Public Libraries: Results of the Burnaby Study. Paper presentado en Netspeed Conference, Calgary, AB.

Cyberbullying: el acoso a través de las redes sociales. Recuperado el 12 de septiembre de 2016. Disponible en http://www.revistacabal.coop/actualidad/cyberbullying-el-acoso-traves-de-las-redessociales

Donnerstein, E. y Smith, S. (2001). Sex in the Media: Theory, Influences and Solutions. En D. Singer y J. Singer (Eds.). Handbook of children and the media (pp. 289-307). Thousand Oaks, CA: Sage.

La tecnología en la preadolescencia y adolescencia: Usos, riesgos y propuestas desde los y las protagonistas. 2010. Save The Children,

El uso de la tecnología para perpetrar la violencia hacia las mujeres. 2010. Recuperado el 3 de noviembre de 2016. Disponible

en www.apc.org/sites/default/files/FactSheetVAWICTs_ES_0.pdfcombatirla

Picaso Mesén M. ¿Cómo podría delinearse una evaluación cualitativa? Ciencias Sociales. 2002 97(3). Recuperado el 12 de junio de 2016. Disponible en: http://www.revistacienciassociales.ucr.ac.cr/wpcontent/revistas/97/04-PICADO_47-61.pdf

Redes Sociales. 2014. Internet 2.0. Observatorio Tecnológico del Ministerio de Educación, cultura y deporte.

Serrano Barquín1 C, Serrano Barquín R. 2014. Ciberacoso en estudiantes universitarios: diagnóstico y medidas correctivas para intervenir en la violencia de género expresada en redes sociales. Revista de Comunicación de la SEECI. Número extraordinario, 94-101 ISSN: 1576-3420 
Dom. Cien., ISSN: 2477-8818

Vol. 3, núm. mon., agos., 2017, pp. 137-148

Cyberbullying: el acoso a través de las redes sociales en jóvenes universitarios

Verdejo Espinosa M A. 2015. Ciberoacoso y violencia de género en redes sociales. Universidad Internacional de Andalucía 